\title{
MAGNETIC ALLOY LOADED RF CAVITY FOR BARRIER BUCKET EXPERIMENT AT THE AGS*
}

\author{
$\underline{\text { M. Fujieda }}^{\dagger}$, Y. Iwashita and A. Noda, Kyoto University, Gokanosho, Uji 611, JAPAN \\ Y. Mori, C. Ohmori and Y. Sato ${ }^{\ddagger}$, KEK-Tanashi, 3-2-1 Midori-cho, Tanashi 188, JAPAN \\ M. Yoshii, KEK, 1-1 Oho, Tsukuba 305, JAPAN \\ M. Blaskiewicz, J. M. Brennan, T. Roser, K. S. Smith, R. Spitz and A. Zaltsmann, \\ BNL, Upton, NY 11973, USA
}

\section{Abstract}

A magnetic alloy(MA) loaded cavity which was developed by KEK was used for the barrier bucket experiment at the AGS. The MA loaded cavity could generate a single sine-wave of $40 \mathrm{kV}$ with less $\mathrm{RF}$ power than a ferrite loaded cavity because of its broad-band impedance. In order to reduce the voltage induce by beam, the feedforward system was applied and the beam loading was compensated. With the barrier bucket scheme, five bunches were transferred successfully. Total of $3 \times 10^{13}$ protons were accumulated without beam loss. They were re-bunched by the accelerating cavities and accelerated.

\section{INTRODUCTION}

A Magnetic Alloy(MA) loaded cavity[1, 2] has been developed. The cavity was installed in the AGS for the barrier bucket experiment. It was the first experiment for the MA loaded cavity to be tested in synchrotron. The beam loading and the method to cure it were important issues. The cavity and the RF amplifier worked stably. The beam loading compensation system was established.

The barrier bucket experiments [3, 4] were performed to increase the intensity at the AGS. Two barrier cavities were used to generate a fixed barrier and moving one. Both cavities generated a single sine-wave of $40 \mathrm{kV}$ at the repetition rate of $351 \mathrm{kHz}$. The former is the MA loaded cavity, the latter is a ferrite loaded cavity modified by BNL. This paper describes the MA loaded cavity and the barrier bucket experiment.

\section{BARRIER CAVITY}

\subsection{MA Loaded Cavity}

The peak current to generate a single sine-wave for a LCR circuit on resonance is represented as follows,

$$
I_{\text {peak }}=\frac{V}{R}(1+Q)
$$

If the impedance is given, the system with lower $Q$ value can generate a barrier voltage with less RF power. The KEK barrier cavity is loaded with MA cores, FINEMET. The cores have large inductance and low $Q$ value. A pushpull amplifier is suitable to drive an MA loaded cavity be-

\footnotetext{
* Work supported by Department of Energy

† Email: miho@tanashi.kek.jp

$\ddagger$ also : Japan Steel Works Co. Ltd.
}

cause the waveform of the drive signal is bipolar.

The impedance seen by beam is $3.6 \mathrm{k} \Omega$ and the $\mathrm{Q}$ value is 0.6. Accordingly the MA loaded cavity has large R/Q as $6 \mathrm{k} \Omega$. The voltage induced by high intensity beam is not negligible and a compensation is necessary for the cavity.

The cavity has 4 gaps and it generates a barrier voltage of $10 \mathrm{kV}$ per gap. Two $30 \mathrm{~kW}$ tetrodes are used for the RF power amplifier[5]. A $2 \mathrm{~kW}$ solid-state amplifier is employed for the drive amplifier. An arbitrary function is applied for the drive signal. Figure 1 shows the barrier voltage and the RF bucket.
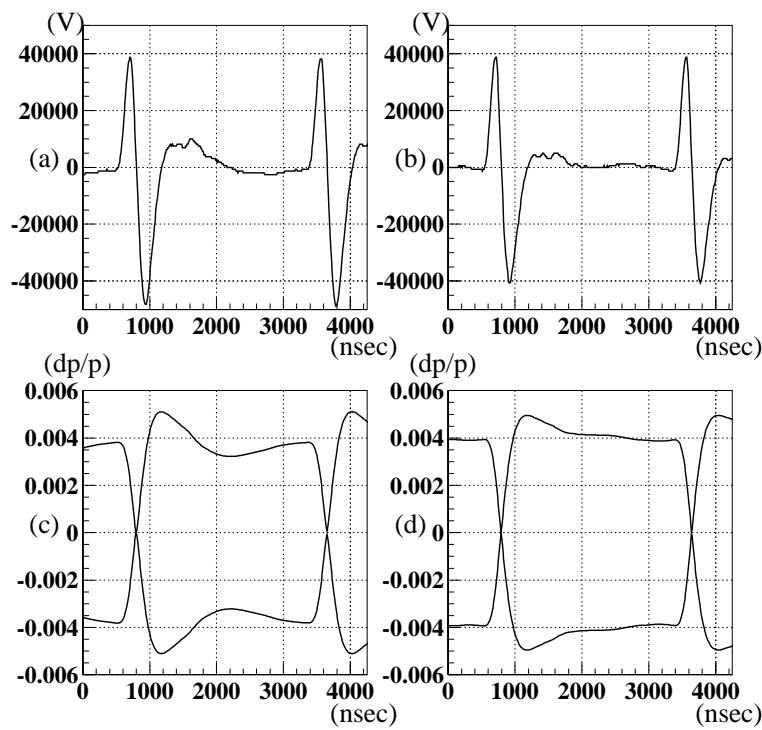

Figure 1: The gap voltage(a),(b) and the RF bucket(c),(d). The waveform(a) was used in the experiment. The waveform(b) was improved to achieve a flatter RF bucket(d) by a drive signal modification and the overshoot is expected to disappear by mixing several harmonics.

\subsection{Feedforward System}

If the beam signal is fed to the broad-band RF amplifier, the beam induced voltage can be canceled[6]. The feedforward[7] scheme was applied for the compensation of the beam loading. The frequency responses of the beam monitor, cables and the grid circuit of the amplifier are important because the $\mathrm{Q}$ value is low and $\mathrm{R} / \mathrm{Q}$ is large. The cavity is suitable for the feedforward scheme, because all parameters are stable against the temperature rise or the beam loading. It is easy to add a compensation signal, because the time response of the cavity to the signal is fast. 
Figure 2 shows the schematic feedforward system. The beam signal was picked up by the wall current monitor. The signal was split into three because of the frequency dependence of the grid circuit and the cable used for delay. Then they were filtered by three types of the filters: low-pass $(\mathrm{h}=1)$, band-pass $(\mathrm{h}=2)$ and high-pass $(\mathrm{h} \geq 3)$ filters. The phase and amplitude of each Fourier component of the gap voltage were adjusted independently. The signal was delayed for one revolution period after the beam passed the cavity. It was because the feedforward path was longer than the beam traveling time from the monitor to the cavity.

The induced voltages per gap with or without the feedforward are shown in Figure 3. It can be seen that the amplitude of the induced voltage decreases to $1 / 9$ effectively. The system worked stably.

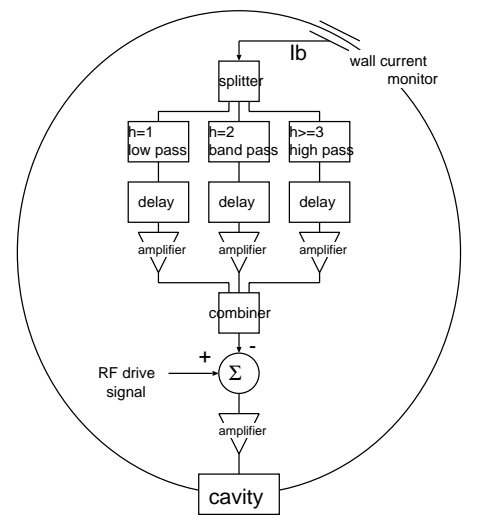

Figure 2: The feedforward system. The revolution frequency is $351 \mathrm{kHz}$.

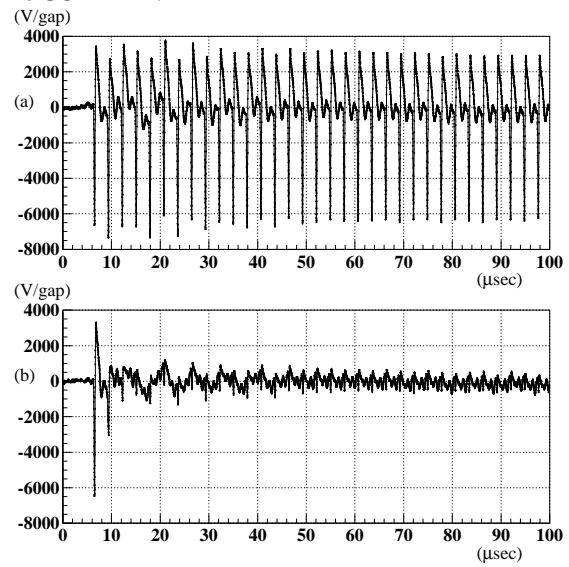

Figure 3: The induced voltages per gap without(a) or with(b) feedforward. The horizontal axis is time. The vertical one is voltage per gap. The beam intensity was $8.2 \times 10^{12}$ protons per bunch and one bunch was circulating.

\section{BARRIER BUCKET}

\subsection{RF Gymnastics}

The barrier cavity has to play two important roles during injection with barrier bucket. The first barrier create a flat RF potential in the longitudinal phase space to keep the beam gap. The second one performs RF gymnastics to accept and accumulate the injected beam. The BNL and KEK cavities took parts of the former and latter, respectively.

One of the barriers is moved to expand the injected beam. This debunch method with using barriers is called as "adiabatic debunch". After debunching, the moving barrier is turned off. It is turned on again at the same phase as the fixed barrier and moved to make the space for next injection. The next bunch is injected into the longitudinal space. Then it debunched adiabatically by the moving barrier, while the stored beam is compressed. The moving barrier is turned off when their momentum spreads become equal. Thus the stored beam and the injected one merge. If those processes are repeated adiabatically, the total emittance of bunches is conserved and beam can be injected as much as the limit of the longitudinal acceptance.

The RF gymnastics were optimized not to cause beam loss and leakage from RF bucket. Figure 4 shows the gymnastics of barriers in case of transfers of five bunches.

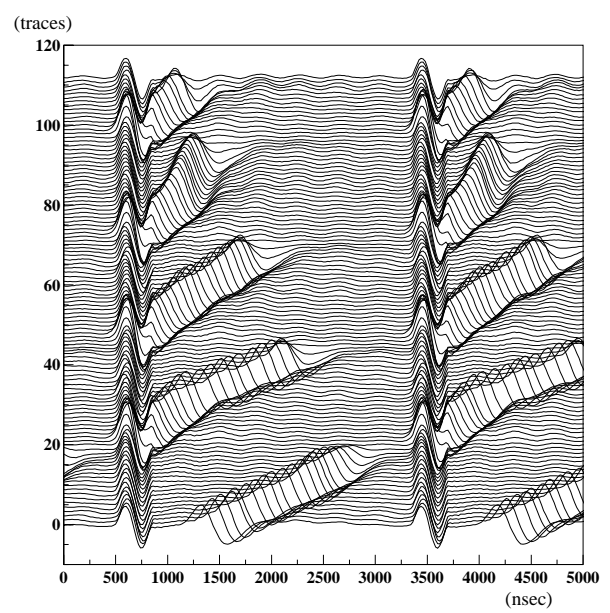

Figure 4: The mountain range plot of the barrier voltages. The interval of the traces is $5.8 \mathrm{msec}$.

\subsection{Adiabatic Debunch}

The sweep speed of a barrier should be slow enough to satisfy the adiabatic condition to conserve the emittance. The time for sweep, however, is limited by the AGS Booster cycle of $150 \mathrm{msec}$

The adiabatic condition was studied measuring the momentum spread by Schottky signal, to determine the longitudinal emittance. The measurement was done varying the barrier speed with one transfer. The initial phase gap between two cavities was $77^{\circ}$ which was wider about 3 times than the bunch width. The phase gap was spread to $315^{\circ}$. The result is shown in Table 1.

In case of the sweep time longer than $100 \mathrm{msec}$, the minimum momentum spread was achieved. The results agree with the fact that if the barrier is swept as slow as 10 times of the debunching time, the momentum spread after debunch is minimized. The longitudinal emittance grew $2 \sim 3$ times. It has been considered to be mainly due to the longitudinal mismatch. The potential distortion also seems to relate with it. 
Table 1: The momentum spread by "adiabatic debunch".

\begin{tabular}{|c|c|c|}
\hline \multirow{2}{*}{$\begin{array}{c}\text { sweep time } \\
(\mathrm{msec})\end{array}$} & \multicolumn{2}{|c|}{ momentum spread $(\%)$} \\
\cline { 2 - 3 } & $\left(\frac{d p}{p}\right)_{0}=0.30 \%$ & $\left(\frac{d p}{p}\right)_{0}=0.44 \%$ \\
\hline 0 & 0.30 & 0.44 \\
25 & 0.24 & \\
50 & 0.16 & \\
100 & 0.13 & 0.11 \\
150 & 0.13 & \\
300 & 0.12 & \\
\hline
\end{tabular}

\subsection{Spacing}

The initial RF bucket should be matched with the bunch width not to cause the longitudinal mismatch. On the other hand, it should be wide enough to avoid that the injection kicker disturbs the circulating beam. The initial phase gap between the barriers was optimized observing beam loss. There was no beam loss by the kicker when the phase gap was wider than $770 \mathrm{nsec}$, which was consistent with the kicker pulse width. However this phase gap width was much wider than the typical bunch width of 200nsec, which should have degraded the emittance. Actually when the phase gap was narrow, smaller emittance was achieved.

\subsection{Re-bunch and Acceleration}

Total of $3 \times 10^{13}$ protons were accumulated successfully. The beam intensity of the first bunch was $6.4 \times 10^{12}$ protons per bunch. After five bunches were transferred, the gaps of the barrier cavities were shorted and the accumulated beam debunched freely. The beam was re-captured by the accelerating cavities of the harmonic number of 6 , which voltage was ramped up linearly. After the re-capture it was accelerated. There was a beam loss at the gamma jump because of the large longitudinal emittance. The emittance after re-bunching by harmonics of 6 was $5.5 \sim 7.0 \mathrm{eV}$ s per bunch, which grew about 3 times. During the re-bunching and the acceleration, no emittance growth nor beam loss was observed. Figure 5 shows the accumulation of 5 bunches and re-bunching.

\section{CONCLUSION}

An MA loaded cavity has been developed for the barrier bucket experiment. It can generate a single sine-wave of $40 \mathrm{kV}$ by the $60 \mathrm{~kW} \mathrm{RF}$ amplifier. The feedforward system to compensate the induced voltage worked successfully. The cavity was suited to barrier bucket due to its operational flexibility.

The barrier bucket experiment has been accomplished with both the MA loaded cavity and the modified AGS RF cavity. The RF gymnastics with the two barrier cavities was established and worked well. The five bunches were transferred. Total of $3 \times 10^{13}$ protons were accumulated without beam loss. Then they were re-bunched and accelerated by the accelerating cavities even though the longitudinal emit-

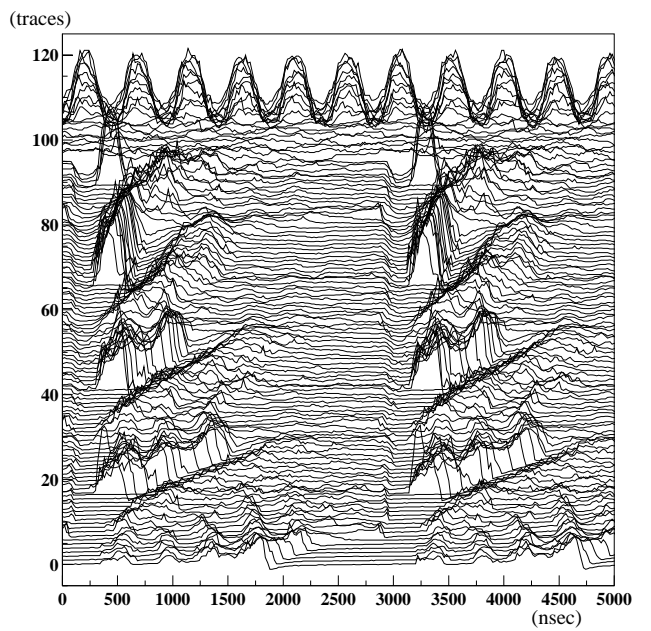

Figure 5: The mountain range plot of the wall current monitor. The interval of their traces is $5.8 \mathrm{msec}$.

tance grew. Following reasons are considered to produce the emittance growth.

- Longitudinal mismatch

- Potential distortion

- Snap off of the barrier by the high Q cavity

The first one seems be major reason. The improvement of the injection kicker will be helpful to decrease the emittance growth caused by the longitudinal mismatch. A special barrier gymnastics also enables to reduce the mismatch. The KEK barrier distorted the RF potential with its overshoot in the waveform. The overshoot has almost disappeared by mixing several harmonics for the drive signal[8] and this scheme can be applied to the cavity. The feedback or feedforward system will help to cancel the induced voltage at the BNL barrier cavity. Amplitude modulation of the BNL cavity will also reduce emittance growth.

\section{ACKNOWLEDGMENT}

We would like to thank all members of AGS and KEKTanashi RF group for their great helps.

\section{REFERENCES}

[1] Y. Mori et al., Proc. of EPAC 98, p1796, Stockholm, (1998).

[2] M. Fujieda et al., Proc. of APAC 98, Tsukuba, (1998).

[3] M. Blaskiewicz et al., in this conference.

[4] M. Blaskiewicz and J. M. Brennan, Proc. of EPAC 96, p2373, Barcelona, (1996).

[5] Y. Sato et al., Proc. of APAC 98, Tsukuba, (1998).

[6] Y. Hashimoto et al., Proc. of EPAC 98, p1770, Stockholm, (1998).

[7] P. Barratt et al., Proc. of EPAC 90, p949, Nice, (1990).

[8] C. Ohmori et al., in this conference. 